\title{
Humans, Animals, and Robots: A Phenomenological Approach to Human-Robot Relations
}

\author{
Mark Coeckelbergh
}

Accepted: 22 August 2010 / Published online: 4 September 2010

(c) The Author(s) 2010. This article is published with open access at Springerlink.com

\begin{abstract}
This paper argues that our understanding of many human-robot relations can be enhanced by comparisons with human-animal relations and by a phenomenological approach which highlights the significance of how robots appear to humans. Some potential gains of this approach are explored by discussing the concept of alterity, diversity and change in human-robot relations, Heidegger's claim that animals are 'poor in world', and the issue of robot-animal relations. These philosophical reflections result in a perspective on human-robot relations that may guide robot design and inspire more empirical human-robot relations research that is sensitive to how robots appear to humans in different contexts at different times.
\end{abstract}

Keywords Human-robot relations · Human-animal relations $\cdot$ Phenomenology $\cdot$ Appearance $\cdot$ Robot-animal relations

\section{Introduction}

The introduction of robots in the personal sphere-now and in the near-future-gives rise to new personal relations between humans and robots. How can we better understand these relations from a philosophical perspective?

The idea of personal relations between humans and robots often invokes science-fiction images of robots that appear human and that are used as substitutes for humans. Ro-

A first version of this paper has been presented at the $2 \mathrm{nd}$ International Conference on Human-Robot Personal Relations, Tilburg, June 11-12, 2009.

M. Coeckelbergh ( $\varangle)$

University of Twente, Enschede, The Netherlands

e-mail: m.coeckelbergh@utwente.nl bots may be presented as partners, as in the film The Stepford Wives (2004), or in other substitute roles, as in Bicentennial Man (1999) and I, Robot (2004). These images do not entirely belong to the domain of fiction: some humans might engage in relationships with human-like robots [1] and as humanoid robotics develops further other practices will emerge. However, it is more likely that in the near future relations between humans and robots will mainly take the form of strong attachments to robots that do not appear human (although they might have some humanoid aspects). They may be given an animal-like appearance (zoomorphic) or a different appearance. Many current robots are zoomorphic: consider for example the iCat (Philips), Huggable (MIT Media Lab), and the 'huggable animal-like robot' Probo (Free University Brussels) [2].

A turn away from anthropomorphism is more likely for several reasons. First, because human appearance is not required it is easier to design the robots and make them available. Second, they avoid the problem of the so-called 'uncanny valley'. The Uncanny Valley hypothesis suggests that if a robot looks too much like a human (but when it is still clear that it is not a human), it appears 'uncanny' [3]. Feelings of uneasy do not occur when the robot looks very different or when we encounter a human; only the appearance of the nearly-human has this effect. Robots that look differently do not incur this problem. Third, since humans already have relations with non-robotic non-humans such as animals and - to some extent - computers and other electronic equipment such as mobile phones, we can expect that the introduction of non-humanoid robots will be less controversial than, for instance, humanoid sex robots. While the account developed here is relevant to relations with humanoid robots, I am also concerned with relations with other robots, in particular intense, long-term relations with non-humanoid 
robots such as those that may develop between children and their toy robot or between adults and pet robots.

In this paper, I propose to understand such relations by taking a phenomenological approach and by comparing human-robot and human-animal relations. I support my argument by drawing on insights from philosophy of technology and by briefly exploring the phenomenology of animals. I show how this approach can enhance our understanding of human-robot relations.

When I use the term 'phenomenology' in this paper I refer to (1) a general orientation that gives attention to how robots appear to human consciousness in order to better understand human-robot relations (hermeneutics rather than science), and (2) two specific currents within the philosophical tradition of phenomenology: the work of Martin Heidegger and the 'postphenomenology' of the American philosopher of technology Don Ihde.

First, I use Ihde's work to characterize personal humanrobot relations as alterity relations and show how this concept could contribute to existing studies of human-robot interaction. Then I discuss the importance of appearance for understanding that interaction and call attention to the variety of human-robot relations in different contexts. I make a comparison between human-robot and human-animal relations, considering in particular robots that represent animals. I respond to Heidegger's work to discuss the relevance of phenomenological similarities and differences between humans, animals, and robots for human-robot relations. Finally, I raise the issue of robot-animal relations.

\section{Human-Robot Relations as Alterity Relations}

In philosophy of technology, Ihde developed a (post)phenomenological framework to discuss human-technology relations [4]. Applying the concept of intentionality to humantechnology relations, he distinguishes three structural features of technological intentionality: embodiment relations, hermeneutic relations, and alterity relations. Let me explain these relations and apply the concepts to robots.

Embodiment relations refer to the amplification of bodily perception: technology comes to be experienced as being part of us; we do no longer notice it. For instance, usually I do not notice that I wear glasses. Most robots will not invite an embodiment relation. Exceptions are perhaps particular non-autonomous robots such as robot suits (for instance HAL), robot arms, or robot 'exo-skeletons' (see for example the performances of the Australian performance artist Stellarc): such robots might come to be experienced as part of the body.

The hermeneutic relation refers to the role technology plays as an in-between that allows us to interpret the world $[4,5]$. We do not have direct access to the world; instead, the technology is between us and the world. Consider the role played by scientific instruments such as telescopes and microscopes: our perception is always mediated by these technologies. For example, research at the nano level is impossible without such mediations. If we had only our human eyes, we could neither see any nano particles nor manipulate them. The technology that shows things at that level is indispensable, not as a mere 'instrument' but as a way of seeing (and manipulating) the world. There are at least two senses in which robots could fulfil such a role. First, they can mediate between us and the world in a rather literal sense, for instance we can imagine a disabled or even locked-in person using a robotic device to give her information and to do things in the world. Or we can think of remote controlled robots on other planets that enable us to see and manipulate the world through the eyes of the robot.

However, the third concept, alterity relations, is the most interesting for the purpose of understanding relations to personal robots: sometimes we relate to technology as an other. What does this mean? Using Ihde's framework, we can understand personal human-robot relations as alterity relations if and to the extent that in these relations appear to humans as an other or 'quasi-other' [6]. The robot is neither part of me (embodiment relation) nor something that mainly mediates my relation to the world. Instead, in our interaction with the robot 'it' appears to us as more than a thing: an other to which I relate [6, p. 107].

This approach to the status of the robot stands in contrast to most traditional philosophical accounts (including traditional phenomenology), which would require that the robot has intentionality, consciousness, and so on. Instead I argue here and elsewhere that what matters for understanding and evaluating human-robot relations is how the robot appears to us. Therefore, even if - based on traditional accounts - we are not prepared to ascribe to robots the character of alterity, we can speak of quasi-alterity in those cases where the robot appears to us as an other. Whatever the 'real' status of the robot may be, it is its appearance that is relevant to how the human-robot relation is experienced and constructed. (I will continue this argument in the next section.)

Moreover, to view relations with robots in this way does not imply that there is a reciprocal relationship of 'selves' or that we need to seek recognition from the 'android partner', as Ramey suggests in his arguments for a phenomenological approach to the ethics of human-android interaction [7]. Moreover, while I sympathize with Ramey's rejection of scientism and with his recommendation that we attend to possible changes in the (human) self as a result of interaction with android robots, I propose that we understand the robot other as an other that is experienced as external. However much truth there may be in Ramey's Heideggerian claim ${ }^{1}$ that we

\footnotetext{
${ }^{1}$ Ramey is influenced by Olafson's interpretation of Heidegger [8].
} 
ek-sist, stand out, it means that we can stand back from our selves and our relations with others and quasi-others, but it does not imply that selves incorporate others (other humans or other entities). In contrast to human-robot embodiment relations, which might involve incorporation into the self, this does not happen in the human-robot relations under consideration here. The robot other appears as an other (although not perhaps as a radical other) and not as part of our selves. This is a condition for having an alterity relation in the first place: we do not incorporate the robot in our perception; instead, the robot appears to us as an other different from ourselves and similar to other (human) others.

This application of the concept of alterity as a way to understand human-robot relations is in line with some findings from existing studies of human-robot interaction. We can compare human-robot relations with other technological alterity relations such as relations with automata (historical) and computers (contemporary). Turkle has argued that we experience computers as being on the border between inanimate and animate [1,9]. More, we do not only experience them as living, we also treat them as such. In particular, sometimes we treat them as we would treat a human other. For example, when the computer 'does' something wrong, we might swear at it as we would do at a human other. We treat it as if it were living and even as if it were human. If this is true for computers, it is all the more applicable to robots operating in the personal sphere: they cross this inanimateanimate border easily - they often appear animate - and we interact with some robots as if they were human. If computers can already give us the experience of animation, we can expect robots to do a much better job: many (autonomous) robots appear animate and whether or not their appearance is human-like, they can be equipped with more possibilities of expression in order to enhance the experience of animacy. Studies indicate that the more animate the face of the robot, the more likely it is to attract human attention and to facilitate human-robot interaction; how people perceive robots is crucial (see for example an experiment by Bartneck et al. [10]). For instance, the robot Probo has facial expressions in order to act as a social interface [2] and earlier Breazeal has already developed the robot Kismet to study interactive social exchange between humans and robots [11-13]. Moreover, human-robot interaction sometimes develops into a (longer-term) human-robot relation. Interestingly, a human-robot relation developed in which Breazeal became a kind of 'mother' of the robot. Other robots are often explicitly designed to take on the role of 'children' or 'babies'. If designers were to make robots that are even more human-like in the way they interact with us, we can expect that more of such human-like relations develop.

\section{Appearance, Diversity, and Change}

This raises the question concerning the importance of appearance for how we relate to robots. If robots appear animate, what is - with regard to personal relations - the relevant difference with an animal, which $i$ s animate by definition? Of course we usually make a distinction: animals are 'natural', 'biological' entities whereas robots are 'artificial', 'technological' objects. But in the particular kind of humanrobot relations under consideration, this difference becomes less relevant. If an alterity relation develops by which the robot appears as a quasi other, then from an appearance point of view there is no fundamental difference between the animal and the robot. Differences are to be studied at the level of appearance: what matters for the human-robot relation is how the robot appears to human consciousness [14]. Ontological differences - what the entity really is - become irrelevant to the development of the human-robot relation as an alterity relation.

A phenomenological analysis of human-robot relations, then, must distinguish between various kinds of humanrobot relations and must do so on the basis of appearance, that is, the appearance of the robot as experienced by the human. Thus, for the purpose of understanding human-robot relations we must distinguish between male and female robots, humanoid robots and pet robots (dogs, cats, etc.), 'friendly' robots versus 'neutral' or 'unfriendly' ones, ${ }^{2}$ etc. Regardless of what the robot 'really' is (if it makes sense to say this at all), in each case, the nature of the human-robot relation will differ, depending on appearance. For instance, we can expect that a person's response to gender differences in relations with robots will resemble that person's response to human gender differences. Another example: we can expect different responses if a researcher were to point out to users or to designers that a particular cleaning robot appears to be modelled after a cleaning lady. Appearance matters, whatever the intention of the designers. And if someone hates (biological) dogs, that person is unlikely to engage in relations with robot dogs-regardless of the ontological difference between the two entities.

These hypotheses are not trivial in the light of a philosophical tradition that attaches much importance to ontological difference. They urge philosophers to turn away from questions such as 'When does a robot have consciousness?' (which concerns the 'mind' of the robot) and to take an approach that is in line with research on how humans perceive and treat new media and robots. Philosophers and robot designers could learn from and inform such research. For example, entirely in line with Turkle's research mentioned above, Reeves and Nass [15] have shown that people

\footnotetext{
${ }^{2}$ Robotics researchers are already trying to give robots 'personalities'.
} 
tend to respond to new media such as computers as they interact with other humans, in a natural and social way. Again it turns out that in this respect, we often do not make a difference between the reality and appearance. And it goes further than shouting at a computer. For instance, people might treat computers as teammates or attribute a gender and personality to them.

Often these effects are unintended. But of course designers can try, and have tried, to imitate humans on purpose. Some of us might see such designs as attempts to 'deceive' or 'trick' people (an interesting issue which I discuss elsewhere). But whether or not this is true, humanoid robotics can be a great tool for enhancing both our scientific knowledge and our philosophical understanding of not only human-robot relations but also human-human relations. In humanoid robotics, Ishiguro has used humanoid robots to study human cognition and interaction (see for example Ishiguro $[16,17])$. Now this is only possible in the first place since we tend to treat humanoid robots as social others. They appear to us (to the human consciousness) as more than things; they become quasi-others as we engage in alterity relations with them. ${ }^{3}$ Thus, if we build human-like robots that have the capacity to appear as quasi-others, we can study humans as social beings and reflect on the origin, development, and meaning of social relations in human contexts as well.

Having said that, interaction with robots does not always go as smoothly as my interpretation of studies such as those offered by Reeves and Nass might suggest. Surely not all human-robot interactions trigger the kind of processes that create and sustain human-robot alterity relations. For instance, as I already mentioned in the beginning of this paper, sometimes humanoid robots may appear 'uncanny'. The Uncanny Valley Hypothesis suggests that subtle differences in appearance (as compared to real humans) make seem certain human-like robots eerie [18]. This renders it possible to use humanoid robots to study the mechanisms involved in evaluations of human likeness [19]. However, those of us who interact or will interact with robots on a daily basis in the near-future are more likely to interact with robots that are not uncanny at all, for instance robot pets. In all these cases, what matters with regard to how we respond to robots is not what robots are but how they appear to usregardless of the technical requirements that render this appearance possible and regardless of the ontological status ascribed to the robot. But we should keep in mind that appearances can change. For instance, more information about the robot ('it cannot really do this') might change how the

\footnotetext{
${ }^{3}$ Note that consciousness is interpreted here in a non-dualist way: rather than an absolute distinction between mind and world, consciousness is seen here as bound up with our engagement with the world: our (inter)actions, our relationships, etc.
}

robot appears to us and weaken the human-robot relation or even completely erase its alterity aspect. Thus, human-robot relations must be viewed as dynamic processes with might or might not bear the mark of alterity at particular times or periods of time.

\section{Robots and Animals}

So far, I sketched an account of human-robot relations as alterity relations that centres on the importance of appearance with its aspects of diversity and change. In order to further develop this view, let me draw on the analogy with human-animal relations. For a start, the animal does not exist. It may serve well as a category for other purposes, but when it comes to understanding human-animal relations it is not very helpful. There are many kinds of animals, they play different roles, and hence our relation to them differs as well. Ethological studies [20] and pragmatist literature on animal ethics [21] highlight the variety of human-animal relations. We have different relations with animals in different contexts: animals can appear as companions (pets), as 'living meat', livestock, and production units (farm animals), as game (animals we hunt), as 'wild' animals (in our Romantic imagination), as experimental material (in scientific experiments), and as entertainment (e.g. animals in the zoo). Appearance-in-context (among other things) explains this difference in human-animal relations, not only the (ontological) features of the animal as defined by science. The same animal may even appear differently in different contexts. Consider how in some societies and cultures dogs are perceived as potential food whereas in our society this would be seen as an outrageous thing to do; dogs are generally perceived as companions, slaves, and so on. Pigs and rats share a lot with us biologically speaking, but we treat most of them as raw materials for our meat production and experimental research. In a different context they might also be used as pets or feature in movies as cuddly animals. This suggests that the appearance of the animal (as related to personal preference, context, and other factors), not the ontological status, matters with regard to how we relate to them. And appearance depends on (our human) perception, which depends on personal, contextual, and cultural categories. To return one of the examples provided above: in a farm context, most animals are treated differently than in a home context: rather than being considered and treated as companions, they appear to us as 'living meat', a resource (consider the term livestock). To use a Heideggerian term: they are part of the Gestell (usually translated as 'enframing'): we engage in a way of thinking and perceiving that lets appear nature as a reserve [22]. Our collective frame of mind encourages viewing animals as a reserve we can use for our purposes: to feed us, to entertain us, to work for us. 
This argument about different contexts and perception can be applied to robots as well: we make differences in treatment according to appearance in different contexts. For instance, if certain robots appear to us as not cuddly, they are likely to be treated badly or at least we will not form strong and warm attachments to them. And compare an industrial context (robots as slaves) with a home entertainment context (robots as companions or pets), where the robots involved look differently and are treated differently. We might also consider cultural differences between views about humanrobot relations and the different traditions of thought that use different conceptual categories and have a different history of robot perception. For instance, there seem to be differences between Europe and Japan in the way robots are perceived: the 'slave' model seems to be more dominant in Europe whereas Japan seems to go for the 'companion' model. Of course one should be cautious about making such generalizations-for example, it is clear that cultures and societies are not homogenous and it is a matter of discussion how significant differences are and how they are to be observed or measured - but the point here is that what matters for how human-robot relations take shape are not the ontological features of the robot but how the robot appears to us, humans. Surely this depends on features of the robot as well, but these are always features-as-perceived-by-us, by the subject. They are not essential characteristics which allow straightforward conclusions about the human-robot relation; how they are viewed depends on the social and cultural context in which the relation develops. It could imply, for example, that in some parts of the world humans are not inclined to develop alterity relations with robots since they are used to make a sharp distinction between humans and non-humans, subjects and objects, persons and things, and so on, whereas in other parts of the world such dualisms are not entrenched in the culture and do not inhibit the development of alterity relations: the robot then appears as an other, not a 'mere thing'. In sum, contextual differences are crucial to the way robots appear to humans and therefore to how human-robot relations are shaped. These differences can be related to differences within a society between functions or domains (food, industry, entertainment) and to differences between societies and cultures. More empirical research could reveal other contextual differences and their implications for human-robot relations as dependent on appearance.

Note that if I am right about the importance of appearance for human-robot relations, one may well ask if there are relevant differences between a 'real' robot and a virtual robot when it comes to understanding what goes on in human-robot relations. For instance, consider a robot in Second Life or a robot in a game. How material must a robot be in order for us to engage in alterity relations with that robot? I suggest that since what matters from a phenomenological perspective is appearance, strong experiences of otherness of virtual robots and intense alterity relations with virtual robots are possible provided that the technological medium delivers a sufficient degree of immersion. For the relation, it does not matter if the robot-or the animal—only exists in the virtual world. The real/virtual distinction seems irrelevant to the conditions under which alterity relations can develop. Even if there is a moderate degree of immersion, robots are likely treated in a 'human' way provided the robot has the right kind of appearance. The immersion need not be total. Film might do. Consider, for instance, people's emotional responses to WALL-E, a little robot which appears as having emotions (for example feeling lonely). The fact that WALL-E only exists on the screen does not prevent people from seeing the robot as an other. Of course, this may not be a lasting alterity relation. As indicated above, relations are dynamic. But the conditions for the development and persistence of the alterity relation will depend on appearance. Thus, this approach is applicable to both 'real' and virtual robots that we might relate to. When we encounter them in our labs, homes, games, virtual worlds, or films, what matters for our relation to them is how they appear to us as subjects-in-context. I have argued that this is analogous to what happens in human-animal relations.

This analogy does not imply that robots and human-robot relations are necessary always and in all possible ways comparable animals and human-animal relations. Robots are not natural entities-they have different 'bodies' and they do not need domestication - and with some robots we may well develop relations that are in several respects different from the kinds of relations we know. But how these relations (will) take shape depends again on appearance and context. As said, this will happen regardless of the natural/artificial distinction. Moreover, as any relation human-robot relations can change and do change. Thus, if at one point in time a particular robot is treated as a slave, we cannot predict that this will be the case in the future. And even within a particular relational 'model' there is plenty of room for variety and change. For example, there are considerable differences between persons; how we interact with robots and the precise form of our relation with them partly depends on our own character, identity, and personal history. As in human-animal relations, the relation we develop with robots depends on our first contact(s) and further experiences with particular robots. As Waiblinger argues about humananimal relations: 'the history of previous interactions forms the foundation of the current relationship' and 'a relationship is always dynamic' [23]. This historical aspect is often neglected in reflections on human-robot relations.

Given this dynamic, historical dimension of human-robot relations, perhaps there is a sense in which robots need to be 'domesticated': robots designed to be home companions may need to adapt to, and be adapted to, the specific home 
environment in which they are used: they have to adapt to the physical space of the house, to the particular people and their routines, etc. Moreover, if human-like, social robots (robots that are able to live together with humans) were to become better at learning, they would need to be 'educated', to be 'tamed' in a way somewhat similar to the training of some pets or young children. They may have a (programmed) basis of abilities but that basis would need to be developed and refined. Even if they never reached the capacity level and status of adult human beings, they would have to develop at least part of their social intelligence by means of a path of domestication and socialization. Moreover, to some extent the robot would also 'tame' the humans: humans would need to adapt to the robot, to get used to it, to become familiar with it. ${ }^{4}$ A similar two-way process happens when animals are introduced into a home environment.

\section{Robots as Animals}

Some robots are explicitly meant to represent or simulate (non-human) animals, for example pet robots or toy robots. To understand this range of human-robot relations, we can learn from research on human-animal relations and engage with context-sensitive animal ethics. I gave a few examples in the previous section. But can phenomenological reflections on human-animal relations help also?

Although there are exceptions [24], this area is not welldeveloped in philosophical phenomenology. Generally relations between humans and animals receive little attention in this tradition. 'Classical' phenomenology stresses the differences (and similarities) between humans and animals. The interest is mainly anthropological, that is, one attempts to define the human by making distinctions between humans and animals. The border between humans and animals is defended by setting up conceptual strongholds along the line. This has prevented systematic and sustained reflections on human-animal relations and, by extension, on any relations between humans and non-humans. Nevertheless, studying and discussing these arguments can be part of trans-disciplinary attempts to understand human-robot relations.

Let me give an example in order to support this claim and to show how the approach offered in this article sheds a different light on the question regarding the difference between humans and non-humans. In his lectures Heidegger argued that, in contrast to humans, animals are 'poor in world' [25]. By this phrase he meant that the animals do not have the

\footnotetext{
${ }^{4}$ This line of reasoning is entirely hypothetical: there may be reasons why building such intelligent, learning social robots is not possible or why their introduction in home environments is undesirable or even unacceptable.
}

range and depth of access to the world human beings have. For Heidegger, an animal behaves towards objects but has no knowledge of them. It is absorbed. It cannot grasp another animal or any other being as a being.

One may object that this is not always true for all animals at all times. Heidegger's reflections on 'the animal' illustrate the neglect of diversity mentioned above. But either way, let us apply Heidegger's argument to robots and suppose that robots seem to have a similar mood (Stimmung) $[25,26]$ as the one Heidegger ascribes to animals, that they can only behave but they do not know. Robots then 'suffer' the same 'deprivation' as animals do according to Heidegger.

One may object to this claim and argue that there are no reasons - in principle - why robots could not have a similar 'mood' or attitude towards the word. But if the Heideggerian claim is true, what follows for understanding human-robot relations? Even if there really was such a fundamental distinction between humans and animals, it need not have implications for human-robot relationships. Consider again the alterity relation. Within the experiential space of this relation, the robot appears as a quasi-other having the same or a similar relation to the world: it appears as a knowing other attuned to the world and to the human other. In other words, it appears to have a mood or attunement similar to humans. (The same is true for animals, of course: whether or not they really have that mood or attunement ${ }^{5}$, there are animals that sometimes appear to have it.) As argued above, what counts for understanding human-robot relations is not the relation the robot may have to the world, but their appearance to us, humans - that is, our relation to others and the world. If this is so, then the 'mood' of the robot (if it could have one at all), is only relevant in so far as it produces a certain appearance, an appearance which does or does not contribute to the development of an alterity relation. If this is true, the question whether or not humans are really or fundamentally different from non-humans such as animals or robots is much less relevant to how we should shape our relations with these non-humans than usually supposed.

\section{Robot-Animal Relations}

Finally, let me raise an issue that is seldom noted: relations between animals and robots. If a robot is introduced into our homes, how will (biological) pets respond (if we have any)? How does and how should a particular robot interact with a cat or a dog and vice versa? Answers to these

\footnotetext{
${ }^{5}$ It is unclear how we could find out at all. This is 'the problem of other minds', which we meet not only in human-human relations but also in human-animal and human-robot relations. A major advantage of the approach proposed here is that this problem is avoided: the question is not what the other really thinks, feels, etc.; instead, the starting point of the analysis is what the other appears to think, feel, and so on.
} 
questions partly depend on empirical observation: we can observe and describe what the introduction of robots does to animals. Studies of the long-term effects of introducing robotic technologies in homes ${ }^{6}$ could be enhanced by including robot-animal relations. But how we anticipate and interpret this and how we think about the normative question (as human subjects) is not only a matter of knowing what kind of robot would be introduced-including knowledge about its appearance-but is also at least partly dependent on our view of animals. If animals are seen as 'pre-programmed' machines (notice the robot-animal analogy), then surely we have a problem: there is no 'build-in programme' that allows the animal to respond to robots. However, animals tend to be far more adaptable to what happens in their environment and respond in ways that cannot be understood by using (simple) machine or robot metaphors. From a phenomenological point of view (applied to the conscious of the animal rather than the human), to understand their response to robots we must attend to the significance of the appearance of the robot to the animal for the robot-animal interaction. For instance, if the robot appears to the animal as a pet of the same 'species', then it is likely that there will be less (unexpected) problems with robot-animal interaction and that alterity-like robot-animal relations will develop, since the animal has already behavioural patterns available to respond to the appearance of the robot. Thus, in this area too phenomenological reflection can generate hypotheses (which then need to be tested by means of empirical research), can make us reflect on animal consciousness, and can contribute to a better understanding of what robot do and will do to our lives and the lives of animals.

\section{Conclusion}

In this paper I have argued that our understanding of many human-robot relations can be enhanced by comparisons with human-animal relations and by a phenomenological approach which highlights the significance of how robots appear to humans and which asks the question if humans are fundamentally different from non-humans. Some potential gains of this approach have been explored by discussing the concept of alterity, diversity and change in human-robot relations, Heidegger's claim that animals are 'poor in world', and the issue of robot-animal relations. Insights gained in these discussions could be used as building blocks for a more systematic appearance-oriented and context-sensitive account of how we should understand various human-robot relations. This is not only interesting for philosophers; it

\footnotetext{
${ }^{6}$ See for example a long-term field study by Sung et al. on what they call 'Domestic Robot Ecology': the holistic and temporal relations that robots shape in their (home) environment [27].
}

also offers a perspective that may guide robot design and inspire more empirical research on human-robot relations and robot-animal relations as dependent on context and robot appearance.

Open Access This article is distributed under the terms of the Creative Commons Attribution Noncommercial License which permits any noncommercial use, distribution, and reproduction in any medium, provided the original author(s) and source are credited.

\section{References}

1. Levy D (2007) Love and sex with robots: the evolution of humanrobot relationships. Harper, New York

2. Saldien J, Goris K, Vanderborght B, Vanderfaeillie J, Lefeber D (2010) Expressing emotions with the social robot Probo. Int J Soc Robot. Available online: doi:10.1007/s12369-010-0067-6

3. Mori M (1970) Bukimi no tani (The uncanny valley). (Trans KF MacDorman \& T Minato). Energy 7(4):33-35

4. Ihde D (1990) Technology and the lifeworld. Indiana University Press, Bloomington/Minneapolis

5. Selinger E (ed) (2006) Postphenomenology: a critical companion to Ihde. SUNY Press, Albany, p 5

6. Ihde D (1990) Technology and the lifeworld, pp 97-108

7. Ramey CH (2005) "For the sake of others": the personal ethics of human-android interaction. In: Proceedings of the android science workshop 2005, cognitive science society. Retrieved May 7, 2009. http://www.androidscience.com/proceedings2005/Ramey CogSci2005AS.pdf

8. Olafson FA (1995) What is a human being? A heideggerian view. Cambridge University Press, New York

9. Turkle S (1984) The second self: computers and the human spirit. Simon and Schuster, New York

10. Bartneck C, Kanda T, Mubin O, Al Mahmud A (2009) Does the design of a robot influence its animacy and perceived intelligence? Int J Soc Robot 1:195-204

11. Breazeal C (2002) Designing sociable robots. MIT Press, Cambridge

12. Breazeal C (2003) Emotion and sociable humanoid robots. Int $\mathbf{J}$ Hum-Comput Stud 59(1-2):119-155

13. Breazeal C (2003) Toward sociable robots. Robot Auton Syst 42:167-175

14. Coeckelbergh M (2009) Personal robots, appearance, and the good: a methodological reflection on roboethics. Int J Soc Robot 1(3):217-221

15. Reeves B, Nass CI (1996) The media equation: how people treat computers, television, and new media like real people and places. CSLI Publications, Stanford. Cambridge University Press, New York

16. Ishiguro $H$ (2006) The uncanny advantage of using androids in cognitive and social science research. Interact Stud 7(3):297-333

17. Ishiguro $H$ (2006) Android science: conscious and subconscious recognition. Connect Sci 18(4):319-332

18. Mori M (1970) Bukimi no tani (The uncanny valley)

19. MacDorman KF (2005) Androids as an experimental apparatus: why is there an uncanny valley and can we exploit it? In: Proceedings of the android science workshop 2005, cognitive science society. Retrieved May 7, 2009. http://www.androidscience.com/ proceedings2005/MacDormanCogSci2005AS.pdf

20. Waiblinger W (2009) Human-animal relations. In: Jensen P (ed) The ethology of domestic animals, 2nd edn. CAB, Wallingford/Cambridge, $\mathrm{p} 102$ 
21. Harbers H (2002) Weak ethics, strong feelings. In: Keulartz J, Korthals M, Schermer M, Swierstra T (eds) Pragmatist ethics for a technological culture. Kluwer Academic, Dordrecht, pp 143-149

22. Heidegger M (1977) The question concerning technology. In: The question concerning technology and other essays (Trans Lovitt W). Harper and Row, New York. 1953

23. Waiblinger W (2009) Human-animal relations, p 104

24. Lotz C, Painter C (eds) (2007) Phenomenology and the nonhuman animal: at the limits of experience. Springer, Berlin, pp 1328

25. Heidegger M (1995) The fundamental concepts of metaphysics (Trans McNeill W, Walker N). Indiana University Press, Bloomington, p 195 (1929/1930)

26. Kuperus G (2007) Attunement, deprivation, and drive: Heidegger and animality. In: Lotz C, Painter C (eds) Phenomenology and the non-human animal: at the limits of experience. Springer, Berlin, pp 13-28

27. Sung JY, Grinter RE, Christensen HI (2010) Domestic robot ecology. Int J Soc Robot. Available online: doi:10.1007/s12369 $-010-0065-8$

Mark Coeckelbergh teaches philosophy of technology at the Philosophy Department of the University of Twente, The Netherlands. He published Liberation and Passion (2002), The Metaphysics of Autonomy (2004), Imagination and Principles (2007), and numerous articles on ethics of technology. His current research focuses on information technology, artificial intelligence, and robotics. 